\title{
Cord blood versus age 5 mononuclear cell proliferation on IgE and asthma
}

\author{
Carolyn Chang ${ }^{1}$, Kevin Gauvey-Kern', Alina Johnson', Elizabeth A Kelvin², Ginger L Chew², Frederica Perera², \\ Rachel L Miller ${ }^{1,2,3^{*}}$
}

\begin{abstract}
Background: Fetal immune responses following exposure of mothers to allergens during pregnancy may influence the subsequent risk of childhood asthma. However, the association of allergen-induced cord blood mononuclear cell (CBMC) proliferation and cytokine production with later allergic immune responses and asthma has been controversial. Our objective was to compare indoor allergen-induced CBMC with age 5 peripheral blood mononuclear cell (PBMC) proliferation and determine which may be associated with age 5 allergic immune responses and asthma in an inner city cohort.

Methods: As part of an ongoing cohort study of the Columbia Center for Children's Environmental Health (CCCEH), CBMCs and age 5 PBMCs were cultured with cockroach, mouse, and dust mite protein extracts. CBMC proliferation and cytokine (IL-5 and IFN- $\gamma$ ) responses, and age 5 PBMC proliferation responses, were compared to anti-cockroach, anti-mouse, and anti-dust mite lgE levels, wheeze, cough, eczema and asthma.

Results: Correlations between CBMC and age 5 PBMC proliferation in response to cockroach, mouse, and dust mite antigens were nonsignificant. Cockroach-, mouse-, and dust mite-induced CBMC proliferation and cytokine responses were not associated with allergen-specific lgE at ages 2, 3, and 5, or with asthma and eczema at age 5. However, after adjusting for potential confounders, age 5 cockroach-induced PBMC proliferation was associated with anti-cockroach IgE, total IgE, and asthma ( $p<0.05)$.
\end{abstract}

Conclusion: In contrast to allergen-induced CBMC proliferation, age 5 cockroach-induced PBMC proliferation was associated with age 5 specific and total lgE, and asthma, in an inner-city cohort where cockroach allergens are prevalent and exposure can be high.

\section{Background}

There has been longstanding controversy in the literature regarding whether biomarkers measured in cord blood may help predict subsequent childhood asthma or atopy [1-9]. Prospective birth cohorts studies have demonstrated that cord blood IgE is a better predictor of skin prick test (SPT) positivity to aeroallergens (dust mite, grass, cat and dog) than family history when assessed up to age 5 years [2,6,7]. A similar positive association with early asthma at age 5 years has been more difficult to demonstrate [2,7]. However, others have shown a positive association between elevated cord

\footnotetext{
* Correspondence: rlm14@columbia.edu

'Division of Pulmonary, Allergy and Critical Care Medicine, Columbia University College of Physicians \& Surgeons, New York, NY, USA
}

blood IgE with risk of later asthma at age 10 years [9], and allergic rhinoconjunctivitis at age 20 years [8].

The findings continue to be mixed when comparing aeroallergen-induced cord blood mononuclear cell (CBMC) proliferation with the subsequent risk for developing asthma, eczema, and allergic rhinitis [10-12]. At birth, infants who developed allergic disease by age 1 year had significantly more positive CBMC responses to dust mite and food allergen proteins than newborns who did not develop allergy [13]. Notably, one birth cohort that followed children as long as 6 years demonstrated no significant difference in aeroallergen-induced (dust mite, grass, mold, cat) CBMC proliferation among cord blood samples of children who subsequently developed atopic disease by 6 years of age compared with samples from children who did not [14]. It has been argued that allergen-induced CBMC proliferation may 
represent a default immune response by recent thymic emigrants as opposed to a more mature $\mathrm{T}$ cell memory response [15]. However, other approaches, such as those using MHC tetramer staining, have demonstrated antigen specific intrauterine $\mathrm{T}$ cell immune response following environmental exposures that display features of immunologic effector memory [16].

Only a few studies have reported on mitogen or antigen-induced CBMC T helper (Th) cytokine production, and compared their levels with the likelihood of later atopy. For example, increased phytohemagglutinin (PHA)-induced interleukin (IL)-5 and IL-13 was associated with increased total IgE during the first year of life [17]. Dust mite-induced CBMC production of IL-13 was associated with SPT positivity in response to dust mite antigens at age 6 [10]. However, associations between dust mite-induced CBMC production of IL-6 and IL-10 and subsequent atopic disease (i.e. asthma, eczema) or SPTs at age 6 years were absent $[10,18]$.

Despite this body of work, studies to date have not yet compared prospectively the association between antigen-induced lymphoproliferative responses in cord blood with repeat measures in later childhood, and assessed the relative strengths of their associations with childhood asthma or eczema. In addition, the roles of early immune responses following ex vivo stimulation with cockroach and mouse proteins, antigens associated with inner city asthma $[19,20]$, have not been fully elucidated. Our objective, using a longitudinal birth cohort designed to examine risk factors for the development of asthma in an inner city population, was to compare cockroach, mouse and dust mite antigen-induced lymphoproliferative response in cord blood with age 5 antigen-specific lymphoproliferative response among the same children, and determine whether either were associated with a greater likelihood of age 5 atopy. We hypothesized that indoor allergen-specific cord blood proliferation and Th2 cytokine production would be associated with subsequent childhood (ages 2, 3, and 5) IgE, asthma, and eczema. We report that, in contrast to allergen-induced CBMC proliferation, age 5 cockroachinduced PBMC proliferation was associated with age 5 specific and total IgE, and asthma, in an inner-city cohort where cockroach allergens are prevalent and exposure can be high.

\section{Methods}

\section{Study subjects}

As part of an ongoing longitudinal birth cohort study conducted under the auspices of the Columbia Center for Children's Environmental Health (CCCEH), women ages 18 to 35, living in Northern Manhattan and the South Bronx, were enrolled during pregnancy $(n=725)$ from clinics affiliated with New York Presbyterian
Hospital (Columbia campus) or Harlem Hospital as described [19,21,22]. Exclusion criteria for pregnant women included smoking, illicit drug use, diabetes, hypertension, HIV infection, and residence in New York City for less than one year.

From this cohort of fully enrolled mothers, a sample based on the number of children from whom a blood sample was obtained (i.e. any time point from cord blood through age 5 years) was selected for inclusion $(\mathrm{n}=609)$. For longitudinal analysis, a subset $(\mathrm{n}=359)$ inclusive of all children for whom cord blood was collected and data were available for prospective analysis at age 2, 3 and 5 year was studied. For cross-sectional analysis, another overlapping subset $(\mathrm{n}=352)$ inclusive of all children for whom age 5 blood was collected was assessed for concurrent (age 5) outcomes symptoms.

Written informed consent was obtained from all study participants and Columbia University's Institutional Review Board approved the study.

\section{Questionnaires}

Detailed questionnaires were administered to women prenatally, every 3 months until the child was age 2 , and every 6 months thereafter until age $5[19,22]$. Questionnaires assessed demographics, maternal asthma, environmental tobacco smoke (ETS) exposure, and report of wheeze, cough and physician diagnosis of asthma and/or eczema. Furthermore, at age 5, parental report of eczema was determined using the validated International Study of Asthma and Allergy in Childhood (ISAAC) eczema questionnaire [23-25], and parental report of asthma was determined using the locally validated Brief Respiratory Questionnaire (BRQ) [26].

\section{Home allergen measurements}

Dust samples were vacuumed separately from kitchens and mothers' beds prenatally and were analyzed for mouse urinary protein (MUP), dust mite (Der $\mathrm{f} 1$ ), and cockroach (Bla g 2) allergens by enzyme-linked immunosorbent assay (ELISA) as described [27-30].

\section{Blood collection, mononuclear proliferation, cytokine assays, and $\lg \mathrm{E}$}

Cord bloods were collected at delivery and maternal blood within 1 day postpartum [19,21,22,31]. Peripheral blood samples at 2, 3 and 5 years of age were collected. Briefly, fresh mononuclear cells were isolated by density centrifugation and plated in triplicate for mononuclear proliferation and in duplicate for cytokine assays. Antigen-induced mononuclear cell proliferation and cytokine production were measured in cord blood and in peripheral blood at age 5 years.

Mononuclear cells $\left(3 \times 10^{5}\right.$ cells/well) were cultured in microtiter plates for 5 days with Blatella germanica 
(German cockroach; $10 \mu \mathrm{g} / \mathrm{ml}$; Greer Laboratories, Lenoir, NC), Dermatophagoides farinae (dust mite; $10 \mu \mathrm{g} / \mathrm{ml}$; Greer Laboratories, Lenoir, NC), Mus musculus (mouse protein extract; $10 \mu \mathrm{g} / \mathrm{ml}$; Greer Laboratories, Lenoir, NC), or no antigen [21]. Increased proliferation in response to German cockroach, dust mite, and mouse protein extract antigens were detected by tritiated thymidine incorporation. Increased mononuclear cell proliferation were defined as (1) a stimulation index [SI] (averaged counts per minute [cpm] in the presence of antigen divided by averaged cpm without antigen) greater than 2, and (2) antigen-induced cpm greater than 1,000 above background [21]. Separate cell aliquots for cytokine analysis were cultured under identical conditions, and supernatants were collected at day 5 and analyzed in duplicates for IL- 5 and IFN- $\gamma$ via ELISA kits (Immunotech, Marseille, France) [21].

Anti-cockroach, anti-mouse, and anti-dust mite IgE levels were measured in sera initially by using the Fluorescence Allergosorbent Test (FAST) (Bio Whittaker, Walkersville, MD) until August 2002. Subsequently, all samples were measured by ImmunoCAP (Phadia, Uppsala, Sweden). Total IgE levels were measured initially by immunoradiometric assay (IRMA) (Total IgE IRMA; Diagnostics Products Corp, Los Angeles, CA), and subsequently (after August 2002) by ImmunoCAP (Phadia, Uppsala, Sweden). All samples were measured in duplicate and during the transition of one validated method to another, a subset was analyzed using both methods to ensure correlation of results, as previously described [19]. Antigen-specific IgE levels of $0.35 \mathrm{IU} / \mathrm{ml}$ or greater (class I) were considered positive.

\section{Statistical analyses}

Data were analyzed with SPSS version 16.0 (SPSS, Inc, Chicago, Ill). Dust allergens levels were analyzed as natural logarithm-transformed continuous values and in tertiles. Mononuclear cell proliferation results were analyzed as continuous (antigen-induced cpm divided by background $\mathrm{cpm}$ ) or dichotomous (positive versus negative SI) variables. Cytokine responses to cockroach, mouse, and dust mite antigens were measured as cytokine ratios: (measured response to antigen)/(measured response to background condition) and analyzed as continuous variables. Allergen-specific IgE levels were analyzed as dichotomous variables $(\geq 0.35 \mathrm{IU} / \mathrm{ml},<0.35 \mathrm{IU} /$ $\mathrm{ml}$ ). Total IgE and sum of allergen-specific IgE (sum of anti-cockroach, anti-mouse, and anti-dust mite IgE) were analyzed as continuous variables. The later approach was intended to study a derived indicator of allergic sensitization to indoor allergens, important to inner city asthma [19], with the benefit of greater statistical power. All values below limit of detection (LOD) were recoded as half LOD. Symptoms and diagnoses assessed by questionnaires were analyzed as dichotomous variables (yes or no). All continuous variables were natural $\log$ transformed.

Fisher's Exact Test and nonparametric tests, including Mann-Whitney U (MWU) Test, Kruskal-Wallis Test, and Spearman's rho correlation, were used to ensure data results were not distorted by failure to fulfill parametric distribution requirements. Unadjusted logistic and linear regression analyses were performed to assess whether allergen-specific mononuclear cell proliferation were significant predictors of atopy and asthma, eczema, allergen-specific IgE levels, and total IgE levels. Adjusted multivariate logistic and linear regression models were examined to adjust for (1) child's sex, (2) ethnicity, (3) any ETS exposure at home at age of interest, (4) maternal history of asthma, and (5) prenatal allergen levels in bed. Interaction terms also were examined between each independent variables of interest and each of the five covariates described above in order to differentiate between confounders and effect modifiers. Statistical significance was defined as a two-tailed $\mathrm{p}<0.05$.

\section{Results}

Study population, age-related indoor antigen-induced proliferation

Children were predominantly Dominican (63.8\%) with lower socioeconomic status and frequent use of public assistance. Twenty one percent of the mothers reported asthma, whereas asthma diagnosis in the child ranged from approximately $15 \%$ to $17.7 \%$ at ages 2,3 respectively, and occurred among $29.6 \%$ by age 5 years (Tables $1,2)$.

\section{Table 1 Characteristics of Study Children}

\begin{tabular}{|c|c|c|}
\hline & $\%$ & $\mathrm{n} /$ Total \\
\hline \multicolumn{3}{|l|}{ Sex } \\
\hline Female & 52.0 & $316 / 608$ \\
\hline \multicolumn{3}{|l|}{ Ethnicity } \\
\hline Dominican & 63.8 & $388 / 608$ \\
\hline African American & 36.2 & $220 / 608$ \\
\hline Maternal History of Asthma & 21.4 & $130 / 608$ \\
\hline Prenatal ETS Exposure* & 49.7 & $237 / 477$ \\
\hline \multicolumn{3}{|l|}{ Postnatal ETS Exposure } \\
\hline Age 2 & 43.5 & $202 / 464$ \\
\hline Age 3 & 49.3 & $225 / 456$ \\
\hline Age 5 & 59.2 & $239 / 404$ \\
\hline \multicolumn{3}{|l|}{ Maternal Highest Degree } \\
\hline High School Diploma or More & 64.9 & $389 / 599$ \\
\hline Currently on Medicaid & 90.3 & $548 / 607$ \\
\hline Currently in Public Assistance & 42.3 & $255 / 603$ \\
\hline
\end{tabular}

Values shown are validated percents to account for missing variables. ETS, environmental tobacco smoke

*In the absence of maternal smoking 
Table 2 Childhood Symptoms at Ages 2, 3, and 5 Years

\begin{tabular}{|c|c|c|}
\hline & Frequency (\%) & $\mathrm{n} /$ Total \\
\hline \multicolumn{3}{|c|}{ Age 2} \\
\hline Asthma $^{1}$ & 15.2 & $41 / 269$ \\
\hline Wheeze ${ }^{1}$ & 15.3 & $41 / 268$ \\
\hline Cough $^{1}$ & 50.2 & $135 / 269$ \\
\hline Eczema $^{1}$ & 21.6 & $57 / 264$ \\
\hline \multicolumn{3}{|c|}{ Age 3} \\
\hline Asthma $^{1}$ & 17.7 & $48 / 274$ \\
\hline Wheeze $^{1}$ & 11.8 & $32 / 272$ \\
\hline Cough $^{1}$ & 47.1 & $128 / 272$ \\
\hline Eczema $^{1}$ & 25.1 & $68 / 271$ \\
\hline \multicolumn{3}{|c|}{ Age 5} \\
\hline Physician Diagnosed Asthma ${ }^{2}$ & 29.6 & $66 / 223$ \\
\hline Wheeze/Whistling/Cough/Other ${ }^{2}$ & 31.8 & $71 / 223$ \\
\hline Cough $^{1}$ & 49.2 & $119 / 242$ \\
\hline Itchy Rash Last 12 months $^{3}$ & 65.9 & $29 / 44$ \\
\hline Ever Had Eczema ${ }^{3}$ & 27.9 & $62 / 222$ \\
\hline
\end{tabular}

Values shown are validated percents to account for missing variables. Sample inclusive of children in whom we have cord blood biological data ( $\mathrm{n}$ $=359$ ).

Parental report of symptoms assessed by: 'Study Questionnaire, ${ }^{2}$ Brief Respiratory

Questionnaire, and ${ }^{3}$ ISAAC Eczema Module.

ISAAC, International Study of Asthma and Allergy in Childhood

Significant correlations between CBMC and age 5 PBMC proliferation in response to all three antigens examined were absent even after stratifying by antigenspecific maternal blood proliferation positivity (Table 3 ). In addition, maternal and cord blood mononuclear cell proliferation continued to differ with each other in response to cockroach as reported in 2001 [21] and now when reanalyzed in 2010 (2001: $\mathrm{n}=133, \mathrm{p}<0.05$ by Fisher's Exact Test; 2010: $\mathrm{n}=277, \mathrm{p}<0.05$ by Fisher's Exact Test). However, statistically differences between maternal and cord blood in the response to mouse antigen that were absent in 2001 were now detected in the larger data set (2001: $\mathrm{n}=132, \mathrm{p}>0.05$ by Fisher's Exact Test; 2010: $\mathrm{n}=252, \mathrm{p}<0.05$ by Fisher's Exact Test). In comparison, statistically significant maternal versus cord blood differences follow exposure to dust mite antigens

Table 3 Correlation of Cord Blood and Age 5 Blood Antigen Specific Proliferation

\begin{tabular}{lcccc}
\hline & & \multicolumn{3}{c}{ Cord Blood } \\
\multirow{2}{*}{ Age 5 Blood } & N & $\mathbf{R}$ & *p-value \\
\cline { 3 - 5 } & Cockroach & 97 & -0.033 & 0.750 \\
& Mouse & 86 & -0.074 & 0.5 \\
& Dust Mite & 88 & -0.171 & 0.111 \\
\hline
\end{tabular}

Analyses by Spearman's rho correlations, $\mathrm{p}$-value two-tailed

$\mathrm{N}$, number of observations

$\mathrm{R}$, correlation coefficient

*Results did not differ after stratifying by antigen-specific maternal blood proliferation positivity. detected in 2001 were not apparent in 2010 with the larger data set (2001: $\mathrm{n}=131, \mathrm{p}<0.05$ by Fisher's Exact Test; 2010: $\mathrm{n}=259, \mathrm{p}>0.05$ by Fisher's Exact Test) [21]. Furthermore, antigen-specific maternal blood mononuclear cell proliferation was not correlated with antigen-specific age 5 mononuclear cell proliferation (cockroach antigen $\mathrm{n}=87, \mathrm{p}=0.82$; mouse antigen $\mathrm{n}=75, \mathrm{p}=0.33$; dust mite antigen $\mathrm{n}=80, \mathrm{p}=0.07 \mathrm{l}$; by Fisher's Exact Test), suggesting that maternal antigeninduced peripheral blood mononuclear cell proliferation does not predict the development of specific antigeninduced $\mathrm{T}$ cell proliferative responses in their children through age 5 years.

\section{Cord blood proliferation, cytokine production, IgE and respiratory symptoms}

By age 5 years, $18.8 \%, 10.8 \%$, and $8.1 \%$ of children developed positive anti-cockroach, anti-mouse, and anti-dust mite IgE, respectively (Figure 1). However, cockroach-, mouse-, and dust mite-induced CBMC proliferation and IL-5 and IFN- $\gamma$ cytokine production were not associated with antigen-specific or total IgE levels at ages 2, 3, or 5 (data not shown), suggesting that antigen-induced $\mathrm{T}$ cell allergic immune responses in CBMC are not associated with a greater likelihood of developing allergen-specific IgE responses in early childhood. To assess prospectively whether antigen-induced $\mathrm{T}$ cell proliferative responses were associated with asthma and eczema symptoms through age 5 years, CBMC proliferation was compared to the frequency of parental report of asthma, cough without a cold, wheeze and eczema. Antigen-specific CBMC proliferation was not associated with maternal report of child asthma, cough, wheeze or eczema at ages $2,3$, and 5 ( $\mathrm{p}>0.05)$.

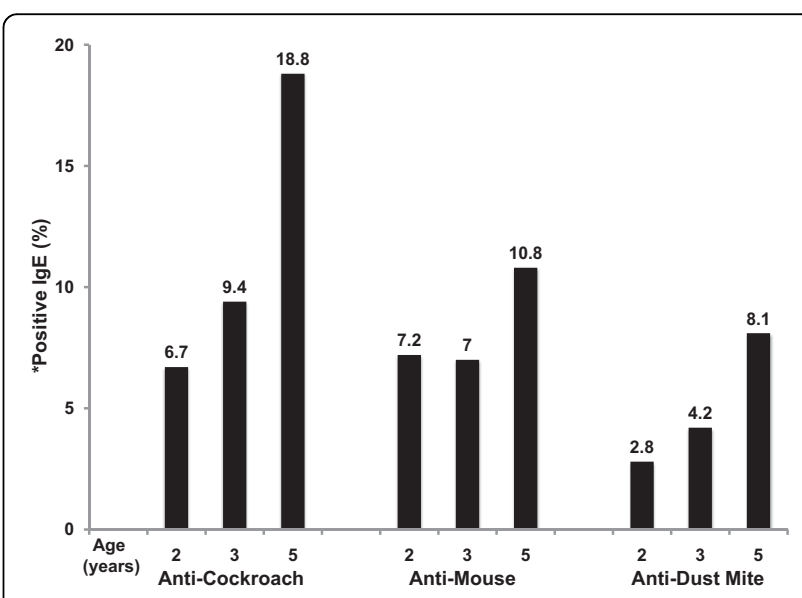

Figure 1 Frequency (\%) of Antigen-Specific IgE Positivity at Ages 2, 3, and 5. *Anti-cockroach, anti-mouse, anti-dust mite lgE positivity is defined as $\geq 0.35 \mathrm{IU} / \mathrm{ml}$. 
Age 5 proliferation, lgE and respiratory symptoms

To ascertain whether concurrent antigen-induced PBMC proliferation is associated with $\operatorname{IgE}$ outcomes at age 5 years, cross-sectional comparisons between age 5 year PBMC proliferation and total and allergen-specific IgE levels were conducted. In univariate analysis, cockroach antigen-induced PBMC proliferation was not associated with anti-cockroach IgE production $(\mathrm{n}=113$, OR 1.75, 95\% CI 0.69, 4.42) (Table 4). However, after adjusting for potential confounders (sex, ethnicity, current ETS exposure, maternal asthma, prenatal cockroach allergen levels in bed), children with positive cockroach-induced PBMC proliferation had almost 3.4 times increased odds of positive concurrent anti-cockroach $\operatorname{IgE}(\mathrm{n}=78$, OR $3.39,95 \%$ CI 1.05, 10.92) compared to children without positive cockroach-induced PBMC proliferation (Table 4). In addition, significant interactions were detectable between age 5 cockroach-induced PBMC proliferation with ethnicity $(\mathrm{p}=0.04)$ and $\operatorname{sex}(\mathrm{p}=0.01)$ on age 5 anti-cockroach IgE. In stratified adjusted models, the associations between cockroach-induced PBMC proliferation and anti-cockroach IgE at age 5 years was detected amongst African Americans $(\beta=1.86, \mathrm{p}=$ $0.00)$ and girls $(\beta=1.05, \mathrm{p}=0.00)$.

Moreover, cockroach antigen-induced PBMC proliferation was correlated weakly with total IgE production at age $5(\mathrm{n}=120$, Spearman's rho $\mathrm{r}=0.225, \mathrm{p}=0.01)$. Cockroach antigen-induced PBMC proliferation was associated significantly with total IgE levels, after adjusting for potential confounders $(\mathrm{n}=85, \beta=1.05, \mathrm{p}=$ $0.00)$ (Table 4). In contrast, mouse- and dust miteinduced PBMC proliferation did not demonstrate significant associations with antigen-specific IgE or total IgE at age 5 years (MWU Test $\mathrm{p}>0.05$ ). Cockroach and mouse allergen levels in bed and kitchen were not associated with antigen-specific CBMC or age 5 PBMC proliferation ratios (Kruskal-Wallis Test $\mathrm{p}>0.05$ ).

Positive cockroach-induced age 5 PBMC proliferation also was associated significantly with the report of physician-diagnosed asthma at age $5(\mathrm{n}=131$, MWU Test $\mathrm{p}=0.03$ ). This finding was confirmed after adjusting for the same potential confounders; age 5 children with positive cockroach-induced PBMC proliferation had three times increased odds of reported physician-diagnosed asthma at age $5(\mathrm{n}=99$, OR $3.08,95 \%$ CI 1.13 to 8.37) compared to age 5 children without increased cockroach-induced PBMC proliferation (Table 5).

In light of research indicating that the development of eczema may be related to antigen-specific $\mathrm{T}$ cell immune responses [14], further cross-sectional comparisons between age 5 PBMC proliferation and prevalence of reported eczema were conducted. In univariate analysis, cockroach antigen-induced PBMC proliferation was associated with eczema at age 5 years $(\mathrm{n}=141$, OR 2.41, 95\% CI 1.11, 5.22), but this association did not persist after adjusting for potential confounders (sex, ethnicity, current ETS exposure, maternal asthma, prenatal cockroach allergen levels in bed) (Table 5). Mouse- and dust miteinduced PBMC proliferation also were not associated with asthma or eczema at age 5. Moreover, concurrent cockroach-, mouse-, and dust mite-induced PBMC proliferation was not associated with cough or wheeze at age 5 (MWU Test $\mathrm{p}>0.05$ for both).

Finally, anti-cockroach, anti-mouse, but not anti-dust mite, IgE was associated significantly with concurrent asthma and wheeze at age 5 years (Asthma $n=281-283$ : anti-cockroach IgE, $\mathrm{p}=0.00$; anti-mouse IgE, $\mathrm{p}=0.01$; anti-dust mite IgE, $\mathrm{p}=0.54$; Wheeze $\mathrm{n}=280-282$ : anti-cockroach IgE, $\mathrm{p}=0.00$; anti-mouse IgE, $\mathrm{p}=0.00$; anti-dust mite IgE, $\mathrm{p}=0.12$ by MWU Test).

Table 4 Age 5 Cockroach Antigen-Induced PBMC Proliferation and IgE at Age 5 years

\begin{tabular}{|c|c|c|c|c|}
\hline \multirow[b]{3}{*}{ Predictors } & \multicolumn{4}{|c|}{ Outcomes } \\
\hline & \multicolumn{2}{|c|}{ Anti-cockroach IgE $\geq 0.35 \mathrm{IU} / \mathrm{ml}$} & \multicolumn{2}{|c|}{ Total IgE (IU/ml) } \\
\hline & $\begin{array}{c}\text { Crude OR } \\
(95 \% \mathrm{Cl}) \\
{[\mathrm{n}=113]}\end{array}$ & $\begin{array}{c}\text { Adjusted OR } \\
(95 \% \mathrm{Cl}) \\
{[\mathrm{n}=78]}\end{array}$ & $\begin{array}{l}\text { Crude } \beta \\
{[n=124]}\end{array}$ & $\begin{array}{l}\text { Adjusted } \beta \\
{[n=85]}\end{array}$ \\
\hline Positive cockroach-induced PBMC proliferation & $1.75(0.69,4.42)$ & $3.39(1.05,10.92)$ & $0.63(p=0.02)$ & $1.05(p=0.00)$ \\
\hline Female sex & NA & $0.64(0.22,1.87)$ & NA & $-0.80(p=0.02)$ \\
\hline $\begin{array}{l}\text { African American ethnicity } \\
\text { (Reference = Dominican) }\end{array}$ & NA & $01.12(0.36,3.46)$ & NA & $0.30(p=0.37)$ \\
\hline Age 5 ETS exposed & NA & $0.83(0.27,2.53)$ & NA & $-0.22(p=0.51)$ \\
\hline Maternal history of asthma & NA & $1.20(0.38,3.77)$ & NA & $0.31(p=0.39)$ \\
\hline Prenatal cockroach allergen in bed ( $\mu \mathrm{g} / \mathrm{g}$ dust) & NA & $0.71(0.46,1.12)$ & NA & $-0.10(p=0.36)$ \\
\hline
\end{tabular}

Results were based on univariate and multivariate, logistic and linear regression models. The endpoint anti-cockroach lgE is shown as odds ratio, and total lgE as regression coefficients with two-tailed $p$-values. Interaction terms each examined in a separate model [data not shown] demonstrated significant interactions between age 5 cockroach-induced PBMC proliferation with ethnicity $(p=0.04)$ and sex $(p=0.01)$ on age 5 anti-cockroach IgE. In stratified adjusted models, there were significant positive associations between age 5 cockroach-induced PBMC proliferation and age 5 anti-cockroach lgE amongst African Americans ( $\beta=1.86$, $p=0.00)$ and girls $(\beta=1.05, p=0.00)$. Maternal asthma was defined as positive at either prenatal or 3 -months postnatal time points. Cockroach prenatal allergen levels ( $\mu \mathrm{g} / \mathrm{g}$ of dust) are expressed as natural log-transformed values. OR, odds ratio. ETS, environmental tobacco smoke. NA, not applicable. 
Table 5 Cockroach Antigen-Induced PBMC Proliferation and Asthma, Eczema at Age 5 Years

\begin{tabular}{|c|c|c|}
\hline \multirow[b]{2}{*}{ Predictors } & \multicolumn{2}{|c|}{ Outcomes } \\
\hline & $\begin{array}{c}\text { Asthma }^{1} \\
{[n=99]} \\
\text { OR }(95 \% \mathrm{Cl})\end{array}$ & $\begin{array}{c}\text { Eczema }^{2} \\
{[n=93]} \\
\text { OR }(95 \% \mathrm{Cl})\end{array}$ \\
\hline Positive cockroach-induced PBMC proliferation & $3.08(1.13,8.37)$ & $2.09(0.76,5.75)$ \\
\hline Female sex & $0.35(0.13,0.92)$ & $1.26(0.47,3.37)$ \\
\hline $\begin{array}{l}\text { African American ethnicity } \\
\text { (Reference = Dominican) }\end{array}$ & $2.13(0.81,5.63)$ & $1.28(0.48,3.40)$ \\
\hline Age 5 ETS exposed & $1.36(0.51,3.61)$ & $1.93(0.69,5.40)$ \\
\hline Maternal history of asthma & $3.97(1.43,11.02)$ & $3.48(1.27,9.56)$ \\
\hline Prenatal cockroach allergen in bed ( $\mu \mathrm{g} / \mathrm{g}$ dust) & $0.89(0.62,1.27)$ & $0.99(0.70,1.39)$ \\
\hline
\end{tabular}

Results were based on multivariate logistic regression models. Maternal asthma was defined as positive at either prenatal or 3-months postnatal time points. Cockroach prenatal allergen levels ( $\mu \mathrm{g} / \mathrm{g}$ of dust) are expressed as natural log-transformed values. ${ }^{1} \mathrm{BRQ},{ }^{2}$ ISAAC Eczema Module $B R Q$, Brief Respiratory Questionnaire.

ISAAC, International Study of Asthma and Allergy in Childhood.

ETS, environmental tobacco smoke

\section{Discussion}

The major objective of this study was to compare cord blood and age 5 year biomarkers such as indoor antigen-specific proliferation and cytokine production and determine whether either was associated with IgE and symptoms related to atopy and asthma in an inner city prospective cohort. We found that significant associations between antigen-specific cord blood and age 5 proliferation measures were absent. Instead, cockroachspecific proliferation assessed concurrently at age 5 years, but not in cord blood, was associated with asthma and IgE. Our focus on repeat measures and longitudinal assessment of indoor allergen responses important to inner city asthma $[19,21,22]$ is novel.

A possible explanation for the association of age 5 , but not cord blood, cockroach antigen-induced mononuclear cell proliferation with concurrent asthma and atopy may be that cord blood immune responses at birth are less mature or efficient. In support of this possibility is evidence of reduced CBMC proliferation and Th cytokine responses compared to adult responses after ex vivo stimulation with allergens, PHA, lipid A, and peptidoglycan $[32,33]$. Furthermore, there is evidence of impaired function of $\mathrm{T}$ regulatory cells as well as reduced expression and immature phenotype of transcription factor Foxp3, in cord blood compared to adults [32,34]. Also, $\mathrm{T}$ cell epitope mapping demonstrated that in response to allergen, CBMCs lack the fine specificity demonstrated by adult cells [35]. Moreover, Woodfolk et al. demonstrated evidence of differing strengths of $\mathrm{T}$ cell proliferative responses to Trichophyton rubrum, related to changes in $\mathrm{T}$ cell epitope recognition of the immunodominant amino-terminal that occurred over the first 2 years of life, with no change in the peptide recognition pattern after age 20 months [36]. However, it is becoming evident from our work [16] and others' [37] that the fetal adaptive immune system can be highly functional and capable of responding to antigens. Hence, an alternate explanation for the association of antigen-specific age $5 \mathrm{PBMC}$, but not $\mathrm{CBMC}$, proliferation response with age 5 atopic status is that even though initial $\mathrm{T}$ cell priming to aeroallergens occurs across the placenta [38], clinically significant allergic sensitization to inhalant allergens occurs postnatally in early childhood [39]. Cord blood $\mathrm{T}$ cell responses may be specific and functional, but not necessarily committed [11].

The finding that cockroach allergen-induced proliferation at age 5 years is associated with IgE and asthma-related symptoms, as opposed to responses to other indoor allergens, is consistent with substantial research indicating that cockroach allergen is important in the pathogenesis of inner city asthma [40,41]. They also are consistent with evidence that allergen levels in home dust can be associated with allergeninduced proliferation [42]. Our additional finding that anti-cockroach IgE at age 5 years is associated both with asthma and eczema lends further support to the observed association between cockroach allergen exposure, specific allergic immune responses, and risk for asthma and atopy. Importantly, there are regional differences, as demonstrated by Matsui et al., regarding the burden of household mouse allergens on inner-city childhood asthma in Baltimore, Maryland [20]. Similarly, the association between indoor allergen-specific IgE and asthma and eczema was more apparent for dust mite in other cohorts, where dust mites thrive better, in comparison to New York City $[43,44]$. Further, the absence of an association between any indoor antigen-induced proliferation and eczema suggests that indoor allergen-induced $\mathrm{T}$ cell proliferative responses, in contrast to $B$ cell induced responses, may not modulate the risk of developing eczema in an inner city cohort [19]. Notably, significant positive associations between age 5 cockroach-induced PBMC 
proliferation and age 5 anti-cockroach IgE among girls but not boys may be a result of sex specific genetic linkages as demonstrated by other groups $[45,46]$. Nonetheless, given the wide array and repeated immune responses to indoor antigens demonstrated by our group and others [20,40-42], public health interventions directed toward region-specific allergen reduction in the home may have health benefits to all inner-city children.

We acknowledge several limitations to this study. Atopic and respiratory symptom assessments were conducted via standardized questionnaires based upon maternal reporting. Parental report of physician diagnosis of asthma may not be standardized [26], potentially resulting in misclassification. Due to insufficient sample size, we were unable to compare antigen-specific CBMC and age 5 year cytokine responses with clinical outcomes. Furthermore, due to budgetary constraints, we were not able to measure IgE isotype class switching Th2 cytokines IL-4 and IL-13 and their association with IgE levels. In addition, due to repeated analyses, significant statistical interactions in regression models may represent type 1 error. Despite the prospective longitudinal design of the cohort, due to loss to follow-up, many data analyses were cross-sectional. Finally, host characteristics (i.e. genetics) may add to variations in the results as the development of atopy is influenced by genetic, developmental, and environmental factors [47-49].

\section{Conclusions}

In conclusion, in contrast to cord blood, age 5 PBMC cockroach antigen-induced proliferation was associated with anti-cockroach and total IgE production and asthma in an inner-city cohort where cockroach is a prevalent allergen. If corroborated by further studies, this finding lends to potential clinical significance for use of antigen-specific proliferation assays as a biomarker for current, but not future, atopic status in early childhood.

\section{List of Abbreviations}

BRQ: Brief Respiratory Questionnaire; CBMC: cord blood mononuclear cell; CCCEH: Columbia Center for Children's Environmental Health; CPM: counts per minute; ELISA: enzyme-linked immunosorbent assay; ETS: environmental tobacco smoke; FAST: Fluorescence Allergosorbent Test; IL: interleukin; IRMA: immunoradiometric assay; ISAAC: International Study of Asthma and Allergy in Childhood; MUP: mouse urinary protein; MWU: Mann-Whitney U; PBMC: peripheral blood mononuclear cell; PHA: phytohemagglutinin; SI: stimulation index; SPT: skin prick test; TH: T helper

Declaration of Competing interests

The authors declare that they have no competing interests.

\section{Authors' contributions}

CC conceived of the study with RLM, and participated in its design and coordination, its statistical analysis and helped to draft the manuscript. KGK participated in the conduction and coordination of the study and helped to draft the manuscript. AJ participated in the conduction and coordination of the study. EAK participated in its design and its statistical analysis. GLC participated in the design of the study. FP conceived of the study with RLM, and participated in its design and coordination. RLM conceived of the study, and participated in its design and coordination and statistical analysis and helped to draft the manuscript. All authors read and approved the final manuscript.

\section{Acnowledgements}

Funding was provided by: The National Institute of Environmental Health Science (P01ES09600, R01ES008977, R01ES13163), and U.S. Environmental Protection Agency (R827027).

\section{Author details}

${ }^{1}$ Division of Pulmonary, Allergy and Critical Care Medicine, Columbia University College of Physicians \& Surgeons, New York, NY, USA.

${ }^{2}$ Department of Environmental Health Sciences, Mailman School of Public Health, Columbia University, New York, NY, USA. ${ }^{3}$ Department of Pediatrics, Columbia University College of Physicians \& Surgeons, New York, NY, USA.

Received: 3 February 2010 Accepted: 4 August 2010

Published: 4 August 2010

\section{References}

1. Goldstein IF, Perzanowski MS, Lendor C, Garfinkel RS, Hoepner LA, Chew GL, Perera FP, Miller RL: Prevalence of allergy symptoms and total IgE in a New York City cohort and their association with birth order. Int Arch Allergy Immunol 2005, 137:249-257.

2. Edenharter G, Bergmann RL, Bergmann KE, Wahn V, Forster J, Zepp F, Wahn U: Cord blood-lgE as risk factor and predictor for atopic diseases. Clin Exp Allergy 1998, 28:671-678.

3. Ruiz RG, Richards D, Kemeny DM, Price JF: Neonatal lgE: a poor screen for atopic disease. Clin Exp Allergy 1991, 21:467-472.

4. Lilja G, Johansson SG, Kusoffsky E, Oman H: IgE levels in cord blood and at 4-5 days of age: relation to clinical symptoms of atopic disease up to 18 months of age. Allergy 1990, 45:436-444.

5. Bergmann RL, Edenharter G, Bergmann KE, Guggenmoos-Holzmann I, Forster J, Bauer CP, Wahn V, Zepp F, Wahn U: Predictability of early atopy by cord blood-lgE and parental history. Clin Exp Allergy 1997, 27:752-760.

6. Karmaus $W$, Arshad $H$, Mattes $J$ : Does the sibling effect have its origin in utero? Investigating birth order, cord blood immunoglobulin $\mathrm{E}$ concentration, and allergic sensitization at age 4 years. Am J Epidemiol 2001, 154:909-915.

7. Tariq SM, Arshad SH, Matthews SM, Hakim EA: Elevated cord serum IgE increases the risk of aeroallergen sensitization without increasing respiratory allergic symptoms in early childhood. Clin Exp Allergy 1999, 29:1042-1048.

8. Pesonen M, Kallio MJ, Siimes MA, Elg P, Bjorksten F, Ranki A: Cord serum immunoglobulin $E$ as a risk factor for allergic symptoms and sensitization in children and young adults. Pediatr Allergy Immunol 2009, 20:12-18.

9. Sadeghnejad A, Karmaus W, Davis S, Kurukulaaratchy RJ, Matthews S, Arshad SH: Raised cord serum immunoglobulin $\mathrm{E}$ increases the risk of allergic sensitisation at ages 4 and 10 and asthma at age 10. Thorax 2004, 59:936-942.

10. Chan-Yeung M, Ferguson A, Chan H, Dimich-Ward H, Watson W, Manfreda J, Becker A: Umbilical cord blood mononuclear cell proliferative response to house dust mite does not predict the development of allergic rhinitis and asthma. J Allergy Clin Immunol 1999, 104:317-321.

11. Smillie Fl, Elderfield AJ, Patel F, Cain G, Tavenier G, Brutsche M, Craven M, Custovic A, Woodcock A: Lymphoproliferative responses in cord blood and at one year: no evidence for the effect of in utero exposure to dust mite allergens. Clin Exp Allergy 2001, 31:1194-1204.

12. Prescott SL, Macaubes C, Yabuhara A, Venaille TJ, Holt BJ, Habre W, Loh R, Sly PD, Holt PG: Developing patterns of T cell memory to environmental allergens in the first two years of life. Int Arch Allergy Immunol 1997, 113:75-79.

13. Miles EA, Warner JA, Jones AC, Colwell BM, Bryant TN, Warner JO: Peripheral blood mononuclear cell proliferative responses in the first year of life in babies born to allergic parents. Clin Exp Allergy 1996, 26:780-788. 
14. Prescott $S L$, King B, Strong TL, Holt PG: The value of perinatal immune responses in predicting allergic disease at 6 years of age. Allergy 2003, 58:1187-1194.

15. Thornton CA, Upham JW, Wikstrom ME, Holt BJ, White GP, Sharp MJ, Sly PD, Holt PG: Functional maturation of CD4+CD25+CTLA4+CD45RA+ T regulatory cells in human neonatal $T$ cell responses to environmental antigens/allergens. J Immunol 2004, 173:3084-3092.

16. Rastogi D, Wang C, Mao X, Lendor C, Rothman PB, Miller RL: Antigenspecific immune responses to influenza vaccine in utero. $J$ Clin Invest 2007, 117:1637-1646.

17. Neaville WA, Tisler C, Bhattacharya A, Anklam K, Gilbertson-White S, Hamilton R, Adler K, Dasilva DF, Roberg KA, Carlson-Dakes KT, et al: Developmental cytokine response profiles and the clinical and immunologic expression of atopy during the first year of life. J Allergy Clin Immunol 2003, 112:740-746.

18. Prescott SL, Taylor A, King B, Dunstan J, Upham JW, Thornton CA, Holt PG: Neonatal interleukin-12 capacity is associated with variations in allergenspecific immune responses in the neonatal and postnatal periods. Clin Exp Allergy 2003, 33:566-572.

19. Donohue KM, Al-alem U, Perzanowski MS, Chew GL, Johnson A, Divjan A, Kelvin EA, Hoepner LA, Perera FP, Miller RL: Anti-cockroach and antimouse IgE are associated with early wheeze and atopy in an inner-city birth cohort. J Allergy Clin Immunol 2008, 122:914-920.

20. Matsui EC, Eggleston PA, Buckley TJ, Krishnan JA, Breysse PN, Rand CS, Diette GB: Household mouse allergen exposure and asthma morbidity in inner-city preschool children. Ann Allergy Asthma Immunol 2006, 97:514-520.

21. Miller RL, Chew GL, Bell CA, Biedermann SA, Aggarwal M, Kinney PL, Tsai WY, Whyatt RM, Perera FP, Ford JG: Prenatal exposure, maternal sensitization, and sensitization in utero to indoor allergens in an innercity cohort. Am J Respir Crit Care Med 2001, 164:995-1001.

22. Lendor C, Johnson A, Perzanowski M, Chew GL, Goldstein IF, Kelvin E, Perera F, Miller RL: Effects of winter birth season and prenatal cockroach and mouse allergen exposure on indoor allergen-specific cord blood mononuclear cell proliferation and cytokine production. Ann Allergy Asthma Immunol 2008, 101:193-199.

23. Jenkins MA, Clarke JR, Carlin JB, Robertson CF, Hopper JL, Dalton MF, Holst DP, Choi K, Giles GG: Validation of questionnaire and bronchial hyperresponsiveness against respiratory physician assessment in the diagnosis of asthma. Int J Epidemiol 1996, 25:609-616.

24. Asher MI, Montefort S, Bjorksten B, Lai CK, Strachan DP, Weiland SK, Williams $\mathrm{H}$, Group IPTS: Worldwide time trends in the prevalence of symptoms of asthma, allergic rhinoconjunctivitis, and eczema in childhood: ISAAC Phases One and Three repeat multicountry crosssectional surveys. Lancet 2006, 368:733-743.

25. Valery PC, Purdie DM, Chang AB, Masters IB, Green A: Assessment of the diagnosis and prevalence of asthma in Australian indigenous children. $J$ Clin Epidemiol 2003, 56:629-635.

26. Bonner S, Matte T, Rubin M, Sheares BJ, Fagan JK, Evans D, Mellins RB: Validating an asthma case detection instrument in a Head Start sample. J Sch Health 2006, 76:471-478.

27. Chew GL, Perzanowski MS, Miller RL, Correa JC, Hoepner LA, Jusino CM, Becker MG, Kinney PL: Distribution and determinants of mouse allergen exposure in low-income New York City apartments. Environ Health Perspect 2003, 111:1348-1351.

28. Luczynska CM, Arruda LK, Platts-Mills TA, Miller JD, Lopez M, Chapman MD: A two-site monoclonal antibody ELISA for the quantification of the major Dermatophagoides spp. allergens, Der $\mathrm{p} \mathrm{I}$ and Der $\mathrm{fl}$. J Immunol Methods 1989, 118:227-235.

29. Pollart SM, Smith TF, Morris EC, Gelber LE, Platts-Mills TA, Chapman MD: Environmental exposure to cockroach allergens: analysis with monoclonal antibody-based enzyme immunoassays. J Allergy Clin Immunol 1991, 87:505-510.

30. van Strien RT, Verhoeff AP, van Wijnen JH, Doekes G, de Meer GE, Brunekreef $B$ : Der $p$ I concentrations in mattress surface and floor dust collected from infants' bedrooms. Clin Exp Allergy 1995, 25:1184-1189.

31. Boyum A: Isolation of lymphocytes, granulocytes and macrophages. Scand I Immunol 1976, , Suppl 5: 9-15.

32. Schaub B, Liu J, Schleich I, Hoppler S, Sattler C, von Mutius E: Impairment of T helper and T regulatory cell responses at birth. Allergy 2008, 63:1438-1447.
33. Prescott SL, Macaubas C, Holt BJ, Smallacombe TB, Loh R, Sly PD, Holt PG: Transplacental priming of the human immune system to environmental allergens: universal skewing of initial T cell responses toward the Th2 cytokine profile. J Immunol 1998, 160:4730-4737.

34. Ly NP, Ruiz-Perez B, McLoughlin RM, Visness CM, Wallace PK, Cruikshank WW, Tzianabos AO, O'Connor GT, Gold DR, Gern JE: Characterization of regulatory T cells in urban newborns. Clin Mol Allergy 2009, 7:8.

35. Yabuhara A, Macaubas C, Prescott SL, Venaille TJ, Holt BJ, Habre W, Sly PD, Holt PG: TH2-polarized immunological memory to inhalant allergens in atopics is established during infancy and early childhood. Clin Exp Allergy 1997, 27:1261-1269.

36. Woodfolk JA, Platts-Mills TA: Diversity of the human allergen-specific $T$ cell repertoire associated with distinct skin test reactions: delayed-type hypersensitivity-associated major epitopes induce Th1- and Th2dominated responses. J Immunol 2001, 167:5412-5419.

37. Mold JE, Michaelsson J, Burt TD, Muench MO, Beckerman KP, Busch MP, Lee TH, Nixon DF, McCune JM: Maternal alloantigens promote the development of tolerogenic fetal regulatory T cells in utero. Science 2008, 322:1562-1565.

38. Holt PG: Primary allergic sensitization to environmental antigens: perinatal T cell priming as a determinant of responder phenotype in adulthood. J Exp Med 1996, 183:1297-1301.

39. Rowe J, Kusel M, Holt BJ, Suriyaarachchi D, Serralha M, Hollams E, Yerkovich ST, Subrata LS, Ladyman C, Sadowska A, et al: Prenatal versus postnatal sensitization to environmental allergens in a high-risk birth cohort. J Allergy Clin Immunol 2007, 119:1164-1173.

40. Rosenstreich DL, Eggleston P, Kattan M, Baker D, Slavin RG, Gergen P, Mitchell H, McNiff-Mortimer K, Lynn H, Ownby D, Malveaux F: The role of cockroach allergy and exposure to cockroach allergen in causing morbidity among inner-city children with asthma. N Engl J Med 1997, 336:1356-1363.

41. Chew GL, Perzanowski MS, Canfield SM, Goldstein IF, Mellins RB, Hoepner LA, Ashby-Thompson M, Jacobson JS: Cockroach allergen levels and associations with cockroach-specific IgE. J Allergy Clin Immunol 2008, 121:240-245.

42. Finn PW, Boudreau JO, He H, Wang Y, Chapman MD, Vincent C, Burge HA, Weiss ST, Perkins DL, Gold DR: Children at risk for asthma: home allergen levels, lymphocyte proliferation, and wheeze. J Allergy Clin Immunol 2000, 105:933-942.

43. Wickman M, Ahlstedt S, Lilja G, van Hage Hamsten M: Quantification of IgE antibodies simplifies the classification of allergic diseases in 4-year-old children. A report from the prospective birth cohort study-BAMSE. Pediatr Allergy Immunol 2003, 14:441-447.

44. Schafer T, Heinrich J, Wist M, Adam H, Ring J, Wichmann HE: Association between severity of atopic eczema and degree of sensitization to aeroallergens in schoolchildren. J Allergy Clin Immunol 1999, 104:1280-1284.

45. Hunninghake GM, Lasky-Su J, Soto-Quiros ME, Avila L, Liang C, Lake SL, Hudson TJ, Spesny M, Fournier E, Sylvia JS, et al: Sex-stratified linkage analysis identifies a female-specific locus for IgE to cockroach in Costa Ricans. Am J Respir Crit Care Med 2008, 177:830-836.

46. Raby BA, Soto-Quiros ME, Avila L, Lake SL, Murphy A, Liang C, Fournier E, Spesny M, Sylvia JS, Verner A, et al: Sex-specific linkage to total serum immunoglobulin $\mathrm{E}$ in families of children with asthma in Costa Rica. Hum Mol Genet 2007, 16:243-253.

47. Prescott SL, Macaubas C, Smallacombe T, Holt BJ, Sly PD, Holt PG: Development of allergen-specific T-cell memory in atopic and normal children. Lancet 1999, 353:196-200.

48. Schaub B, Tantisira KG, Gibbons FK, He H, Litonjua AA, Gillman MW, Weiss S, Perkins DL, Gold DR, Finn PW: Fetal cord blood: aspects of heightened immune responses. J Clin Immunol 2005, 25:329-337.

49. Litonjua AA, Carey VJ, Burge HA, Weiss ST, Gold DR: Parental history and the risk for childhood asthma. Does mother confer more risk than father? Am J Respir Crit Care Med 1998, 158:176-181.

doi:10.1186/1476-7961-8-11

Cite this article as: Chang et al:: Cord blood versus age 5 mononuclear cell proliferation on IgE and asthma. Clinical and Molecular Allergy 2010 8:11. 